\title{
Wavelet Based Features for Defect Detection in Fabric using Genetic Algorithm
}

\author{
Prajakta A. Jadhav ${ }^{1}$, Prof.M.S.Biradar ${ }^{2}$ \\ ${ }^{I}$ (Department of E\&TC, SCOE, Sudumbare/Pune University, India) \\ ${ }^{2}$ (HOD,Department of E\&TC, SCOE, Sudumbare/Pune University, India)
}

\begin{abstract}
In this paper a new scheme is proposed for Fabric defect detection in textile industry. For this purpose, wavelet transformer is used as feature extractor of coefficients of fabric. These coefficients can defect main fabric image \& indicate defects of fabric textile by optimal subset of these coefficients. For finding a suitable subset Genetic Algorithm is used defect detector. The Shannon entropy is used as evaluation function in Genetic Algorithm. By using two separable sets of wavelet coefficients for horizontal and vertical defects, it was seen that we get better results for defect detection. The advantage of this approach it improves accuracy of fabric defect detection as well decrease computation time.
\end{abstract}

Keywords: Fabric defect detector, Genetic Algorithm, Subset of fabric, Wavelet transformer.

\section{Introduction}

In textile industry, quality control is very important as it directly affects the final product of the industry. To improve quality of textile industry, inspection is very important. In ancient period the inspection in textile industry were doing manually it gains long time for detection \& it become highly expensive due to labor costing. But now inspections are going through automatic inspector machineries. Failure in selection of fabric direct affects on quality of production in textile industry \& manual inspection requires large time for manufacturing of product. To improve inspection, modern computer vision inspection system came into existence. It improves accuracy of finding defects, reduced labor power.

There are numerous reports that show that computer vision based inspection has become very effective in detecting defects in textile industry [1].Automatic inspections is based computer vision method \& inspect the defect automatically. Defect in textile industry can detect by various methods. Research areas in detecting defects are fabric textile, surface of wood, tile, aluminum, distance difference etc [2].Uniform, random or patterned textures are the various areas of detecting defects in fabric textile [3]. In this proposed method fabric defect detection is done by distance difference. In this proposed system wavelet transformer is used as filter \& Genetic algorithm as defect detector. It improves accuracy as well reduce computational time.

The flow of proposed methods is explained in three main parts. First part consists of extracting coefficient through wavelet transform \& obtaining coefficient of subset. In second part the suitable subsets are selected through genetic algorithm. In third part suitable subsets are applied to other images then defects are detected through genetic algorithm.

In first part, the propose system uses wavelet filter for extracting coefficient of subset. This filter puts special information in each quarter of filtered image. The initial image is divided into four parts by two dimensional wavelet transform. The first part is an approximation of initial image and the other parts include components with high frequencies \& low frequencies. By using wavelet transform as filter the wavelet coefficients are obtained. Then genetic algorithm is applied to find suitable subset is obtained. Then defects are obtained by fabric defect detection.

\section{Types of defects in fabric}

Fabric faults or defects are responsible for nearly $85 \%$ of the defects found in the garment industry. There are many kinds of fabric defects.Much of them are caused by machine malfunctions and have the orientation along pick direction (broken pick yarns or missing pick yarns), they tend to be long and narrow. Other defects are caused by faulty yarns or machine spoils. Slubs are often appeared as point defects; machine oil spoils are often along with the direction along the warp direction, and they are wide and irregular.

1. Broken Ends: This defect is caused by a bunch of broken ends woven in the fabric.

2. Broken Picks : In plain woven fabrics, this defect materializes by the presence of two picks in the same shed for a part of the width of the fabric.

3. Float:A float is the improper interlacement of warp and weft threads in the fabric over a certain area.

4. Gout: A gout is a foreign matter usually lint or waste accidentally woven into the fabric.

5.Hole, Cut or Tear : The occurrence of hole, cut or tear which is self explanatory. 
6. Oil or Other Stain-These are spot defects of oil, rust, grease or other stains found in the fabric.

\section{Literature Review}

Wavelet Transform is decomposition of a signal into constituent parts. This decomposition can done by many ways,

1. Fourier series : Harmonic sinusoids;

Single integer index.

2. Fourier transform: non- harmonic sinusoids; single real index.

3. Walsh Decomposition: "harmonic" square waves; single integer index.

4. Kahunen - Loeve decomposition: Eigen function of covariance; single real index.

5. Short - Time FT (STFT): windowed, non-harmonic sinusoids; double index.

Wavelet transform provide waves in frequency \& time domain. Duration of decomposition of signal is limited \& have limited band-pass components. High frequency components have short duration \& have wide band. Whereas, low frequency components have longer duration $\&$ have narrow band.

\section{The Wavelet Transform}

The wavelet transform is used as filter in this proposed system. The wavelet transform uses a family of wavelet function and its associated scaling function used for decomposition of original image into different subbands, providing both frequency \& spatial function.

Wavelet transform analyses the signal by a group of orthogonal functions in the form of $\Psi \mathrm{m}, \mathrm{n}(\mathrm{X})$, that is computed from translation \& dilation of the mother wavelet $\Psi$. These orthogonal functions are shown below,

$\Psi_{\mathrm{m}, \mathrm{n}}(\mathrm{x})=2 \frac{-m}{2} \Psi\left(2^{-\mathrm{m}} \mathrm{x}^{-\mathrm{n}}\right)$

$m$ and $n$ are real numbers in this equation.

Equation $2 \& 3$ are used as analysis \& synthesis formulas respectively.

$$
\begin{aligned}
& \mathrm{C}_{\mathrm{m}, \mathrm{n}}=\int_{-\infty}^{+\infty} f(x) \psi_{\mathrm{m}, \mathrm{n}} \mathrm{n}(\mathrm{x}) \mathrm{dx} \\
& f(\mathrm{x})=\sum_{\mathrm{m}, \mathrm{n}} \mathrm{C}_{\mathrm{m}, \mathrm{n}} \Psi_{\mathrm{m}, \mathrm{n}}(\mathrm{x})
\end{aligned}
$$

Coefficients of this transform are obtained recursively. A synthesis of $j$ is shown in equation 4 .

$$
f(x)=\sum_{k} C_{0, k} \phi_{0, k}(x)=\sum k C j_{+1}, k^{\phi} j_{-D} d_{j+1, k} \Psi_{j+1, k}(x)
$$

Two dimensional wavelet transform is used as filter. The two dimensional wavelet transform divides image into four part .Other parts include components with high frequencies of image \& edge points' shows vertical, horizontal \& diagonal direction. The wavelet transform uses two types of filter, high frequency filter \& low frequency filter. When first order of DWT is applied on image, the image is divided into four part is $\mathrm{I}_{\mathrm{HH}}$, $\mathrm{I}_{\mathrm{LL}}, \mathrm{I}_{\mathrm{LH}} \& \mathrm{I}_{\mathrm{HL}}$. Where, $\mathrm{H}, \mathrm{L}$ is the high \& low bound filter respectively.

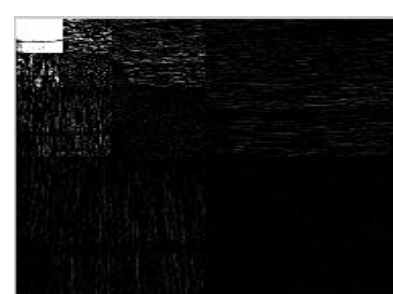

Fig. 1: Original image.

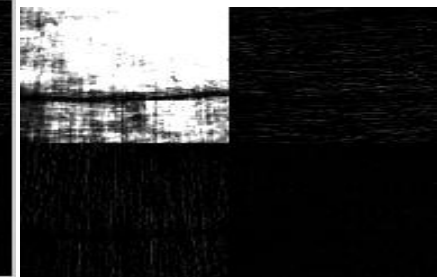

Fig. 2: Wavelet Decomposed image after first order

Wavelet transform evaluates the difference of information in two different resolutions \& creates a multi-resolution view of image's [2]. Hence wavelet transform is used for fabric defect detection. After decomposing image by wavelet filter, the propose system gets coefficients of subset. For finding suitable subset the propose system uses the genetic algorithm. 


\section{The Genetic Algorithm}

In a genetic algorithm, a population of candidate solutions to an optimization problem is evolved towards better solutions [4]. The genetic algorithm is iterative process, the evolution starts from a population of randomly generated individual, with the population in iteration is called a generation.

In each generation the fitness function of every individual is calculated. The fitness function is usually the value of objective function is optimizing the better solution to problem. The more fit individuals are selected from the current population \& each genome of individual is modified for formation of next generation. In next iteration, new generation of candidate solutions is used in next iteration of the algorithm. The algorithm is repeated until satisfactory fitness level is not reached. The generation of second population is done through the genetic operation such as crossover \& mutation.

The simplest form of genetic algorithm involves three types of operators [13]:

1. Selection: This operator selects chromo-somes in population for reproduction. It fitter the chromosome more times so that it is likely to be selected to reproduce.

2. Crossover: The operator in which the subsequences of two chromosomes are exchanged to create new child is called as crossover.This operator roughly mimics biological recombination between two single chromosome organisms.

3. Mutation:This operator randomly flips some bits in a chromosome. Mutuation occur at each bit position in a string with some probability.

The Genetic algorithm work as follows [13]

1. Start with a randomly generated population of N L-bit chromosome.

2. Calculate the fitness function $\mathrm{F}(\mathrm{x})$ of each chromosome $\mathrm{x}$ in the population.

3. Repeat the following steps (a)-(c) until $\mathrm{N}$ offspring have been created:

a. Select a pair of parent chromosome from the current population, with the probability of selection being an increasing function of fitness. Selection is done with replacement," meaning that the same chromosome can be selected more than once to become parents."

b. With probability Pc (the crossover probability) is used to form two offspring. If no crossover takes place, form two offspring that are exact copies of their respective parents.

c. Mutate the two offspring at each locus with probability Pm (the mutation probability), and pace the resulting chromosomes in the new population.

4. Replace the current population with the new population.

5. If the stopping condition has not satisfied, go to step 2 .

\section{Fabric Defect Detection}

In second part, the propose method obtains suitable subset through genetic algorithm after obtaining coefficients of subset by using wavelet transform. To obtain defect in fabric the proposed system has to apply the suitable subset to the images. This suitable subset detects the main image of fabric \& indicates the defects in the fabric image.

If the optimization problem in Equation 5 can be solved, then suitable subset of wavelet coefficient can be

Where,

$$
\text { Min } \mathrm{J}=\mathrm{C}^{*} \mathrm{P} * \mathrm{P}^{\mathrm{T}} * \mathrm{C}^{\mathrm{T}}
$$

$$
\mathrm{C}_{\mathrm{j}(\mathrm{e})}=\sum_{k=0}^{n-1} C_{\mathrm{k}} C_{\mathrm{k}-2 \mathrm{~J}}=0
$$

In this equation $\mathrm{J}$ is the normal form in high frequency components of image, $\mathrm{n}$ is the number of coefficients, $\mathrm{C}$ is subset of wavelet coefficient as a solution, $\mathrm{p}$ is an array of image's pixel values. To minimize $\mathbf{J}$ function through c subset, the proposed system uses the genetic algorithm. In this algorithm, each solution $\mathrm{c}$ is chromosome \& different function can be calculated.

As the proposed system uses the wavelet transform as filter, this filter divides image into vertical \& horizontal directions. Due this it does not give good result when fabric is not symmetric. So, to obtain better result, two separate filter should be used(horizontal \& vertical filter).If W1,W2 is horizontal \& vertical filers \& $\mathrm{u}$ is a perfect image, then filtered image $\mathrm{F}$ can be obtained by,

$$
\mathrm{F}=\mathrm{W} 1 * \mathrm{U} * \mathrm{~W}_{2}^{\mathrm{T}}
$$

In this proposed system the fitness function for filtered image $\mathrm{F}$ is calculated by using sum of squares $\&$ entropy functions. Eq. $7 \& 8$ shows these functions respectively. 


$$
\operatorname{Sum}=\sum_{i=1}^{n} \sum_{j=1}^{m} F(i, j)^{2}
$$

Where,

$\mathrm{n}=$ number of lines,

$\mathrm{m}=$ column in images.

$\&$

$F(i, j)$ is the pixel value of the image, in line umi \& column $j$.

$$
\mathrm{H}=-\sum_{l=1}^{n} d(j) * \log _{2}(\mathrm{~d}(\mathrm{j}))
$$

Where,

$\&$

$\mathrm{n}=$ number of grey levels,

$\mathrm{d}(\mathrm{i})$ is probability of the grey levels in $\mathrm{i}$ in the filtered image $\mathrm{F}$.

This probability is the real number between $0 \& 1$.

With the help of Eq.7 \& 8, optimization problem can be obtained by minimizing $\mathrm{J}$ function in Eq. 9 \& 10 resp.

$$
\begin{aligned}
& \mathrm{J}=\sum_{l=1}^{n} \sum_{j=1}^{m}\left(\mathrm{~W} 1 * \mathrm{U} * \mathrm{~W}_{2}{ }^{\mathrm{T}}\right)^{2} \\
& \mathrm{~J}=\text { Entropy }\left(\mathrm{W}_{1} * \mathrm{U} * \mathrm{~W}_{2}{ }^{\mathrm{T}}\right)
\end{aligned}
$$

The wavelet coefficients are obtained on monotony background of entropy function. So, if the entropy function is very small, the image will be more monotonic. Whereas, sum of square function of image is optimized by minimizing its pixel values. Entropy has simpler computation as compare to sum of squares. So, in this propose system, entropy function is used for calculation of fitness function of Genetic Algorithm.

\section{Expected Result}

In this proposed system, the database of fabric is used for finding defect in it. First, the grey image is converted into contrast enhanced image. After conversion the histogram of image is obtained. This histogram is compared with the histogram of the processed image. After comparison, the wavelet transform is used as filter. In this session, the two dimensional wavelet filter is used. This filter divides the image into four parts $\&$ the coefficients of subsets are extracted through wavelet filter.
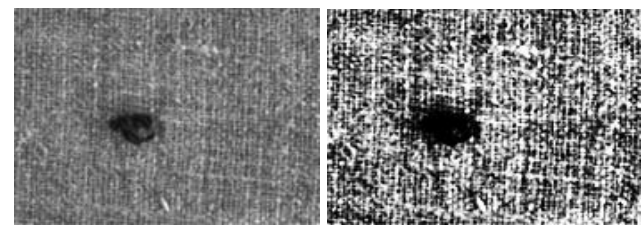

Fig.3 Original grey image Fig. 4 Contrast Enhanced image

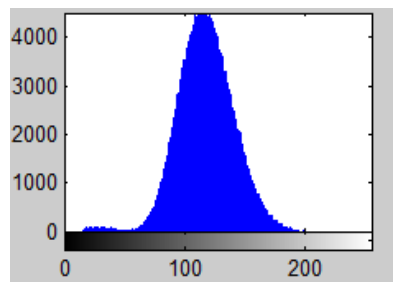

Fig. 5 Histogram of original image

After comprising the histogram with processed image, wavelet decomposition is performed. 


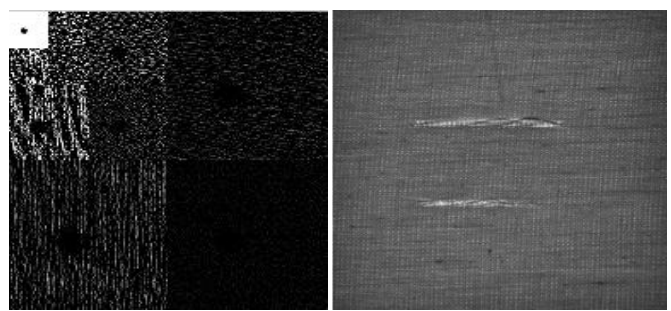

Fig 6. Wavelet Decompose image

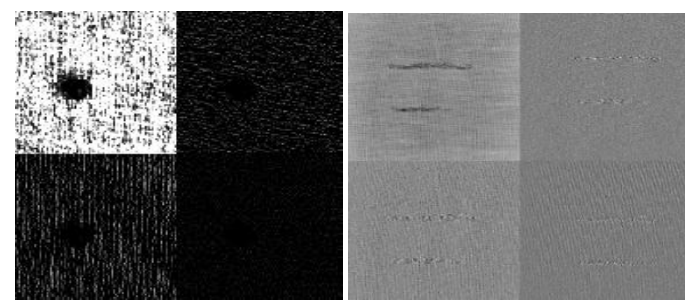

Fig 7. Decomposed image after first stage

After that the proposed system uses genetic algorithm to find suitable subset, then this suitable subset is applied to other images. Original image is deleted $\&$ the defects are gained.

\section{References}

[1] Mahajan P. M., Kolhe S. R and Patil P.M“'A review of automated fabric defect detection".

[2] Narges Heidari, Reza Azmi \& Boshra Pishgoo "Fabric Textile Defect Detection, By Selecting A Suitable Subset of Wavelet Coefficients, through Genetic Algorithm", International Journal of Image Processing (IJIP), Volume (5): Issue (1): 2011

[3] Z. Michalewicz, "Genetic Algorithms + Data Structures = Evolution Programs", AI Series.Springer-Verlag, New York, 3rd edition, (1996)

[4] Lijing Wang, Zhongmin Deng and Xungai Wang "Application of Wavelet Transform Method for Textile Material Feature Extraction".

[5] B.V. Ramana Reddy1, A. Suresh2, M. Radhika Mani3, and V.Vijaya Kumar4 "Classification of Textures Based on Features Extracted from Preprocessing Images on Random Windows", International Journal of Advanced Science and Technology Volume 9, August, 2009.

[6] www.wikipedia.com

[7] Gonzalez R C \& Woods R E, Digital Image Processing, 1982,194.

[8] Image Processing Tool Box for use with Matlab (The Math Works Inc. USA), 2005.

[9] Duda,"Pattern Classification.”Advances in Computational Research, ISSN: 0975-3273, Volume 1, Issue 2, 2009, pp-18-29.

[10] Mahajan P.M.1, Kolhe S.R.2 and Patil P.M.3"A review of automatic fabric defect detection techniques" Bioinfo Publications, Advances in Computational Research, ISSN: 0975-3273, Volume 1, Issue 2, 2009.

[11] Xianghua Xie“A Review of Recent Advances in Surface Defect Detection using Texture analysis Techniques" Electronic Letters on Computer Vision and Image Analysis 7(3):1-22, 2008

[12] K.Srinivasan and P.H. Dastor and P. Radhakrishnaihan, and S. Jayaraman "FDAS: A Knowledge-based Frame Detection Work for Analysis of Defects in Woven TextilStructures", Journal of Textile Institute, vol. 83, pp. 431-447, (1992).

[13] R.Chin,"Automated Visual Inspection Techniques and Applications: A Bibliography”, Pattern Recognition, 15(4): pp. 343-357, (1982).

[14] Z. Guanng and W. Jianxia, "Fabric Defect Detection Method Based on Image Distance Difference", Electronic Measurement and Instruments, (2007), pp. $822-825$.

[15] D. Chetverikov and A. Hanbury, "Finding Defects in Texture Using Regularity and Local Orientation." Pattern Recognition. 35(10), pp. 2165-2180, (2002).

[16] X.Z. Yang and G. Pang and N. Yung, "Discriminative Fabric Defect Detection Using Adaptive Wavelets", Optical Engineering, pp. 3116-3126, December (2002).

[17] X.Z. Yang and G. Pang and N. Yung, "Robust Fabric Defect Detection and Classification Using Multiple Adaptive Wavelets", Image and Signal Processing, IEEE Proceedings, volume 152, PP. 712-723, (2005)

[18] D.Chetverikov "Measuring the Degree of Texture Regularity", In Proceedings of International Conference on Pattern Recognition, 1984, volume 1, PP. 80-82, (1984).

[19] Yu.zhang, zhaoyang Lu, Jing Li,"Fabric Defect Detection \& classification using Gabor filters \& Gaussian Mixture Model", Springer-LNCS, pp: 635-644,(2010).

[20] HAN leil, LI zhong,"quick defect detection based on structure character of woven fabric image \& wavelet transform", computer engineering \& design",(2009)

[21] Rong Fu, Meihong Shi, Hongli Wei, Huijuan chen,"fabric defect detection based on adaptive local binary patterns", international conference on robotics \& bi omimetics, pp: 1336-1340,(2009)

[22] S.Mallat, "A Wavelet Tour of Signal Processing", Academic Press, 2nd ed., San Diego,(1999),

[23] S.Mallat,"A Theory for Multiresolution Signal Decomposition: the Wavelet Representation”,IEEE Transactions on Pattern Anal. and Mach. Intell., vol. 11, no. 7, pp. 674-693, (1989) 